\title{
Augmented Reality and Television: Dimensions and Themes
}

\author{
Pejman Saeghe \\ Department of Computer Science \\ The University of Manchester \\ Manchester, United Kingdom \\ pejman.saeghe@manchester.ac.uk \\ Sarah Clinch \\ Department of Computer Science \\ The University of Manchester \\ Manchester, United Kingdom \\ sarah.clinch@manchester.ac.uk
}

\author{
Gavin Abercrombie \\ Department of Computer Science \\ The University of Manchester \\ Manchester, United Kingdom \\ gavin.abercrombie@manchester.ac.uk \\ Steve Pettifer \\ Department of Computer Science \\ The University of Manchester \\ Manchester, United Kingdom \\ steve.pettifer@manchester.ac.uk
}

\author{
Bruce Weir \\ Research and Development \\ The British Broadcasting Corporation \\ Salford, United Kingdom \\ bruce.weir@bbc.co.uk \\ Robert Stevens \\ Department of Computer Science \\ The University of Manchester \\ Manchester, United Kingdom \\ robert.stevens@manchester.ac.uk
}

\begin{abstract}
Commercialisation of augmented reality (AR) devices has led to their growing application in domestic environments and leisure activities. One such domain is that of television, where AR is one of several technologies driving innovation (c.f. Internet broadcasting, second screen devices). We conduct a systematic literature review to quantify research at the intersection of AR and broadcast television. We identify six common themes and a set of cross-cutting design decisions. We distill this information into a design space incorporating six dimensions: abstraction, interaction, time, display, context and editorial control. We provide methods to operationalise the dimensions to enable research and development of novel concepts, and through this generate six design guidelines to shape future activity at the intersection of AR and television.
\end{abstract}

\section{CCS CONCEPTS}

- General and reference $\rightarrow$ Surveys and overviews; • Information systems $\rightarrow$ Multimedia content creation; • Humancentered computing $\rightarrow$ Mixed / augmented reality.

\section{KEYWORDS}

Augmented Reality; Television; Literature Review; Design Space; Broadcasting; Human-Media Interaction

\section{ACM Reference Format:}

Pejman Saeghe, Gavin Abercrombie, Bruce Weir, Sarah Clinch, Steve Pettifer, and Robert Stevens. 2020. Augmented Reality and Television: Dimensions and Themes. In ACM International Conference on Interactive Media Experiences (IMX '20), fune 17-19, 2020, Cornella, Barcelona, Spain. ACM, New York, NY, USA, 11 pages. https://doi.org/10.1145/3391614.3393649

\section{INTRODUCTION}

Advances in technology lead to changes in broadcast media. This introduces challenges and opportunities for content creators. In

Permission to make digital or hard copies of part or all of this work for personal or classroom use is granted without fee provided that copies are not made or distributed for profit or commercial advantage and that copies bear this notice and the full citation on the first page. Copyrights for third-party components of this work must be honored

For all other uses, contact the owner/author(s)

IMX '20, fune 17-19, 2020, Cornella, Barcelona, Spain

(C) 2020 Copyright held by the owner/author(s)

ACM ISBN 978-1-4503-7976-2/20/06.

https://doi.org/10.1145/3391614.3393649 this paper we seek to provide insights into the design space of a hybrid medium composed of mixing Augmented Reality (AR) with TV.

Various aspects of broadcast media that relate to the production, distribution, and consumption of content, are affected by advances in technology. This in turn affects the viewers' experience. For instance, the introduction of the Internet changed the way the audiences received TV-related data services-from Teletext data embedded in the broadcast signal, to programme specific websites delivered via TCP/IP.

Similarly, recent advances in technologies that enable immersive representations, such as virtual, mixed and augmented reality, have raised the interest of the broadcast and research communities.

There are two ways in which the immersive paradigm can be used: on its own, or combined with another medium. The success of various aspects of the immersive paradigm-as standalone media-may depend on long-term experimentation. These experimentations are often necessary to establish conventions and rules that the content creators implement and the audiences understand. In the meantime, the new medium can be used to remediate [7] existing content and conventions, or in combination with an already established medium such as television.

We select AR from the range of possibilities within the immersive paradigm ${ }^{1}$ and investigate how it can be combined with television to create novel experiences by transforming the conventional TV viewing experience.

Our overall aim is to provide a framework that broadcasters and content creators can use when creating content that mixes conventional TV with AR. We do this by making the following contributions:

(1) A systematic literature review (SLR) that captures publications at the intersection of TV broadcasting and AR between 2008 and 2018;

(2) Identification of six themes (i.e. common motivations and/or problem domains) that emerge from the SLR that can be used to understand the state-of-the-art in the field;

(3) Abstraction of design decisions into a design space composed of six dimensions;

(4) An initial exploration of the design space through (a) two methods of generating 'patterns' that operationalise the

${ }^{1} \mathrm{AR}$ has the potential to become ubiquitous, due to its non-invasive nature. 
dimensions to realise points on the design space, and (b) six practical design guidelines to be considered by content creators when designing novel experiences for the hybrid medium.

When creating experiences that mix AR with TV, content creators are often faced with questions such as:

- What is the relationship between the AR and TV components in such experiences?

- How should the viewers interact with the experience?

- How and when should various components be presented to the viewers?

- What is the role of the viewers' environment in their experience?

We set out to find ways that could be useful in providing answers to questions of this nature and provide concrete guidelines where possible.

Our effort is focused on visual augmentations. Auditory and other sensory augmentations are outside the scope of this paper. We excluded VR from our work, since the broader scope needed to include it in the same study would lead to sacrificing depth for breadth. We suggest that similar investigative work needs to address comparable challenges when combining traditional media (such as TV), with other forms of the immersive paradigm (such as virtual reality).

\section{DEFINITIONS}

We use existing definitions of the terms and concepts in the immersive paradigm and television to explore the characteristics of the emerging hybrid medium that mixes aspects of the two (AR and TV).

Some terms in the immersive landscape have highly standardized definitions (e.g. Virtual Reality), while others have been overloaded with more than one meaning (e.g. Mixed Reality). In such cases, the context in which a term is used may help to disambiguate its intended meaning [56]. For clarity and to provide context we provide widely accepted definitions of key concepts related to this field.

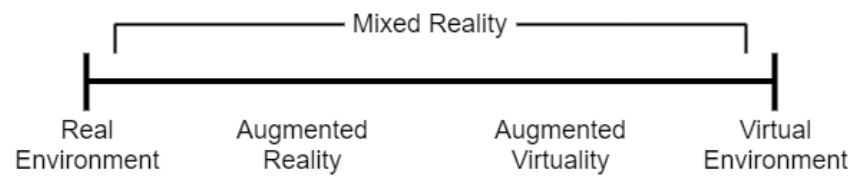

Figure 1: Reality-Virtuality continuum based on Milgram and Kishino's 1994 taxonomy of mixed reality displays.

\subsection{The Immersive Paradigm}

Milgram and Kishino [37] were the first people to present the idea of a continuum to describe this paradigm. Subsequently, Milgram et al. [36,38] referred to this as the Reality-Virtuality continuum (RV).

The real environment and the virtual environment are placed at opposite ends of the RV. Between the two extremes, there are three concepts: Augmented Reality (AR), Mixed Reality (MR), and Augmented Virtuality (AV) (see Figure 1).

Real and Virtual Environments. The real environment refers to a viewer's immediate physical environment. Their view may be direct (e.g. naked eye, see-through glasses), or indirect (e.g. live video feed, pre-recorded video). Conversely, the virtual environment refers to $a$ computer generated model of an imaginary (or a real) environment.

Mixed Reality. Milgram and Kishino referred to any experience that falls between the two ends of RV as mixed reality [37]. However, since then, this term has been used inconsistently. People from neither the researcher nor practitioner communities have an agreed upon definition for this term [56]. MR is often used interchangeably with AR [11].

Augmented Virtuality. This refers to cases where the virtual world dominates the viewers' view of the world, and real objects are used to augment this view.

Augmented Reality. AR was first implemented in 1968 by Ivan Sutherland to display three-dimensional information to the user [57]. It was later defined by Milgram and Kishino to refer "to all cases in which the display of an otherwise real environment is augmented by means of virtual (computer graphic) objects" [37]. Since then, this definition has been revised and refined multiple times.

Azuma defined AR as "a variation of Virtual Environments" which enables "the user to see the real world, with virtual objects superimposed upon or composited with the real world" on the same display [2]. He later suggested three requirements for any AR system: (1) combining "real and virtual objects in a real environment", (2) running "interactively, and in real time", and (3) aligning "real and virtual objects with each other" [1].

In this paper we adopt Milgram and Kishino's [37] definition of $\mathrm{AR}$, since it is the most widely accepted definition [56].

\subsection{Television}

Television was originally conceived in the 1920's as a one-to-many audio-visual successor to radio. It has, however, evolved, benefiting from other technologies and media, in order to improve on how it delivers content and engages with its audiences.

The term TV is overloaded with multiple meanings to reflect various aspects of the industry involved in production, distribution and consumption of audio-visual content. The online English Oxford dictionary defines television as: " a device shaped like a box with a screen that receives electrical signals and changes them into moving images and sound, or the method or business of sending images and sound by electrical signals".

The Internet, as a novel way of distribution, and portable displays, as novel ways of consumption, have transformed the audiences' perception of television and the ways in which TV content is consumed. While many people may not use traditional broadcast channels or use a physical TV set, they may use streaming services (e.g. YouTube and Netflix) and their hand-held displays to watch content that was originally made for TV broadcast by broadcasting corporations (e.g. watching the BBC's The Blue Planet series on Netflix). 


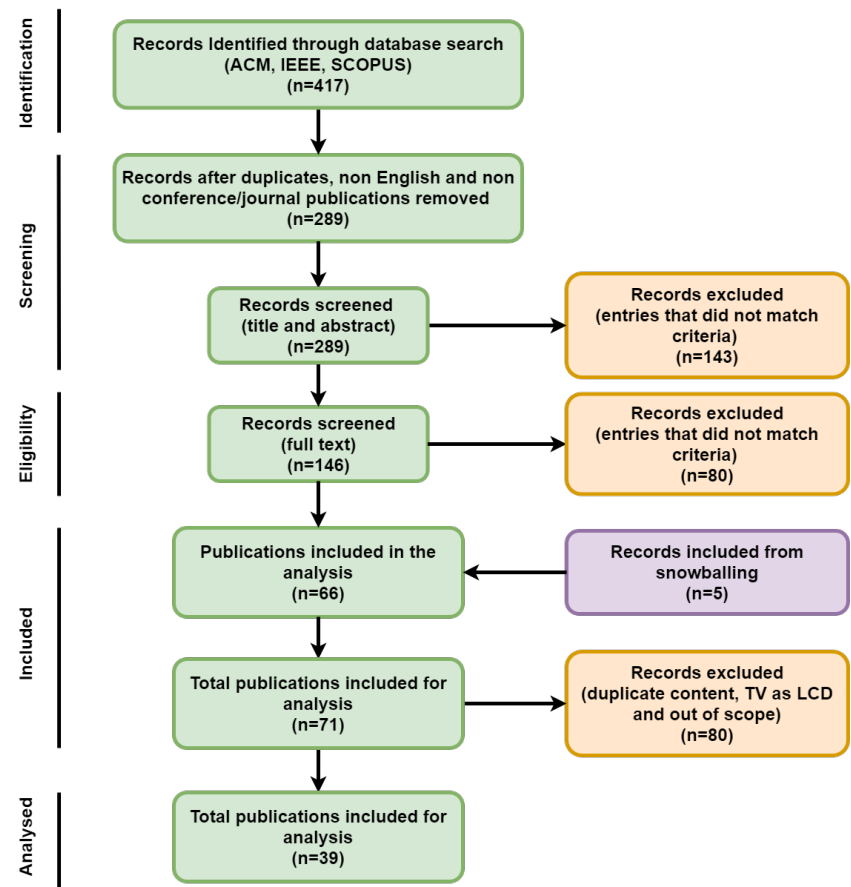

Figure 2: Adapted PRISMA flowchart for selecting publications.

This suggests that, Television should no longer be considered merely to be the physical artefact which is used to display images, but instead as a medium through which a viewer consumes, typically passively, audio-visual content. For example, TV content can be viewed on a physical box in the corner of a living room, streamed to a mobile phone, or rendered onto the side of a building using an AR display.

In this study we include any research that uses the term television (or TV) to describe the work. ${ }^{2}$

\section{METHOD}

Our research is founded on systematic literature review and qualitative analysis methodology. Focusing on recent work, we followed the PRISMA methodology [39] to identify relevant literature published in the 11 years between 2008 and 2018. A representation of the five stages of this process is shown in Figure 2.

\subsection{Paper Selection}

Our initial search terms can be represented as follows:

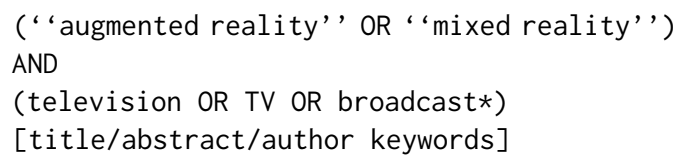

\footnotetext{
${ }^{2}$ Except for when the TV is used as a mere display device.
}

We identified three target databases (SCOPUS, ACM Digital Library $^{3}$, and IEEE) and generated platform-compatible queries for each. Each database search was conducted using the web interface provided. A total of 417 publications were returned by the search (SCOPUS $n=193$, ACM $n=57$, IEEE $n=167$ ).

We excluded duplicates $(n=121)$, papers written in languages other than English $(n=2)$, and results that had not been published as peer-reviewed conference or journal papers $(n=5)$.

The remaining 289 publications were screened by the first two authors, who independently read the titles, abstracts and keywords of the papers. Using the inclusion/exclusion criteria (Table 1), a further 143 papers were excluded.

Table 1: Inclusion/exclusion criteria used to screen the papers returned from the database search.

\section{Inclusion Criteria \\ Returned by one or more target databases \\ Peer reviewed conference/journal paper \\ Uses AR/MR in accordance with widely-accepted definitions [37]. \\ Incorporates some television broadcasting element. \\ Describes concrete scenario or implemented prototype.}

\section{Exclusion Criteria}

Non-English language title/abstract/full text.

Duplicates a system or scenario present in another (more recent) paper in the dataset.

No AR (MR) or no TV present

Posters, talks, courses, panels, demos, white papers etc.

Inter-coder agreement on this task was calculated to be Cohen's kappa $(k)=0.49$, and the authors subsequently resolved all disagreements through discussion.

The same two authors then screened the full texts of the remaining 146 papers, again applying the inclusion/exclusion criteria. This resulted in the removal of a further 80 papers. Agreement at this stage was calculated to be $k=0.85$, and disagreements were once again resolved through discussion.

These screening stages produced a pool of 66 papers. Forward and backward citations were followed in accordance with Wohlin's guidelines for snowballing [63], resulting in the inclusion of five further publications. ${ }^{4}$

A further 14 papers were excluded from this set due to duplicate content, where the same authors described the same concept but published in a different venue. In these cases we kept the most recent publication. A further 18 papers were excluded due to their use of the term $T V$ solely as a display device to show non TV content. This reduced the number of included publications to 39 .

Relying on our knowledge of this area, we added a further three papers that were published within the same time-frame but were not returned by the systematic search [58-60]. These papers were not retrieved due to discrepancies in the terminology used. For

\footnotetext{
${ }^{3}$ A new ACM digital library (DL) was launched publicly in December 2019. Our search was conducted prior to this and thus used the old DL. We have since rerun the query on the new DL and no difference in results was seen

${ }^{4}$ These had not been picked up in the original search as their publishers (Springer Link, Semantic Scholar and the Wiley Online Library) are not indexed by any of the three target databases.
} 
instance, Vatavu [60] used the term augmented $T V$, without mentioning directly the term augmented reality, in the title, abstract, and keywords. The final number of papers analysed was 42 .

Inclusion of MR. We used MR in our query, to ensure the capture of all AR-related papers, since AR and MR are often used interchangeably [11] (see Definitions' Section for further detail).

Table 2: Publications per themes.

$\begin{array}{ll}\text { Theme } & \text { Papers } \\ \text { Living room } & {[3,4,8,20,25-27,29,30,45,46,55,58,59,61,66]} \\ \text { Production } & {[9,13,22,23,28,33,40,50,51,53,54,64,65]} \\ \text { Beyond living room } & {[14,18,41,42]} \\ \text { Remote viewers } & {[31,32,52,60]} \\ \text { Live video } & {[12,15,35]} \\ \text { Photogrammetry } & {[21,49]}\end{array}$

\section{THEMES}

We present six themes that emerge from the qualitative analysis of the publications. These are:

- Enhancing TV Experiences, In The Living Room,

- Production of TV Content,

- Alternative TV Experiences, Beyond Living Room,

- Connecting Remote Viewers,

- Live-Video Augmentation, and

- Photogrammetry.

Table 2 presents the classification of the papers by theme.

Our aim is to identify the themes that emerge from a representative sample of the literature. A comprehensive overview of each theme is outside the scope of this paper.

\subsection{Enhancing TV Experiences, In The Living Room}

This theme covers the largest number of publications in our review $(n=16)$. These studies describe prototypes or scenarios where AR is used to enhance or to transform a traditional TV viewing experience in the living room. We identified three sub-themes that best describe the research in this area: $T V+A R$, focus + context, and $T V$ through $A R$.

$T V+A R$. AR can be used to deliver virtual content $[26,45]$, or virtual screens and controls $[3,58,59]$ outside the physical TV frame.

Focus + Context. The implementation of a focus + context [10] metaphor, allows the viewers to see contextual images in the area around the TV frame. AR can be used in this context to increase immersion $[25,30]$.

TV through AR. AR can replace the physical TV set altogether. By eliminating the conventional TV frame (both physical and virtual) and delivering content that appears to be present in the viewers' environment [66].

\subsection{Production of TV Content}

This theme contains the second largest number of papers in the survey $(n=13)$. Two sub-themes best describe the research in this area: pre-production of TV content, and virtual studio. The main differentiating factor between the two is that the virtual content used in a virtual studio is visible to the viewers at home, whereas the virtual content used in pre-production is ultimately replaced by either real artefacts or higher quality renders.

Virtual Studio. Virtual studio is a mature technology that adds locked-to-camera-view virtual objects to a TV image in real-time. See [19] for an overview. Although researchers often motivated their work by proposing systems for reducing costs and increasing productivity $[9,13,28,54]$ when creating TV content, a virtual studio can also be used to create a production-set which would be physically prohibitive to create.

Pre-Production. In the pre-production phase of content creation, $\mathrm{AR}$ can be used to help directors and set designers visualise a production-set. Using AR, they can place and manipulate various virtual artefacts before committing to a final decision [50]. Alternatively, it can be used during filming to provide a low quality, real-time view of the virtual elements to allow correct shot framing before the creation of the final composition.

\subsection{Alternative TV Experiences, Beyond Living Room}

There are studies that suggest use-cases, at the cross-section of AR and TV that take the TV viewing experience outside the living room. We identified two sub-themes in this category that achieve this in different ways: Cultural Tourism and Film Tourism.

Cultural Tourism. Broadcasters typically own large archives of audio-visual content. This content can often be remediated to create novel experiences using AR. For instance, delivering extra information about a historical landmark using AR [14] or displaying how a landmark looked in certain eras of history. ${ }^{5}$

Film Tourism. Film tourism is defined as tourist visits to destinations as a result of those locations being featured on television or film [24]. AR can be used to enhance film tourism in two ways: by enabling the visitors to watch clips from TV shows that were filmed in important landmarks, when they are vising these landmarks; or by enabling a TV show's fans to follow a specified route and visit multiple filming locations, watching clips from the scenes that were filmed in those locations $[41,42]$.

\subsection{Connecting Remote Viewers}

Television has traditionally been viewed as a medium that brings family and friends together for a shared viewing experience. As the options for viewing content proliferate, there may be fewer opportunities for co-located TV viewing. AR's affordances can be used to simulate a shared viewing experience, for instance, by using virtual avatars [31] or overlaying body silhouettes [60] on the broadcast video.

\footnotetext{
${ }^{5}$ https://www.bbc.co.uk/rd/blog/2019-02-5g-mobile-augmented-reality-bath
} 


\subsection{Live-Video Augmentation}

Advancements in computer vision and related fields can enable the real-time identification and tracking of people and objects present in a live video feed. This information can subsequently be used to overlay virtual content and related information aligned to the people and objects in the scene. In sports broadcasting this can be used to re-texture players' shirts with localized advertisements or statistics [12], or to simulate animated effects performed by the audience of a sports match in a cost-effective way [15].

\subsection{Photogrammetry}

Photogrammetry attempts to obtain "reliable information about the physical objects and the environment through the process of recording, measuring, and interpreting photographic images." 6 In the context of AR and TV, this has been applied to generate 3-D information from 2-D images in order to create a virtual model of the scene [21]. This typically attempts to provide the viewers with a set of options regarding camera angles and ultimately a free-viewpoint viewing experience.

Table 3: Six dimensions of the design space, and their aspects, when mixing AR with TV.

$\begin{array}{ll}\text { Dimension } & \text { Aspect } \\ \text { Abstraction } & \text { Independent; Dependent; Additional AR; Additional TV } \\ \text { Interaction } & \text { Display-level; Structure-level; Content-level } \\ \text { Time } & \text { Continuous; Intermittent; Asynchronous } \\ \text { Display } & \text { Same Display; Separate Displays; TV via AR } \\ \text { Context } & \text { People; Place; Objects } \\ \text { Editorial Control } & \text { Point of View; Sequence }\end{array}$

\section{DIMENSIONS OF THE DESIGN SPACE}

The themes describe the research efforts from a high-level perspective. There are, however, cross-cutting design decisions within the themes that can be further distilled. We present these as six primary dimensions that emerge from a qualitative analysis of the publications.

Our motivation is to enable further exploration of the design space in order to help the content creators and to identify underresearched areas.

The dimensions are:

- the level of abstraction between AR and TV,

- the nature and level of viewers' interaction with the experience,

- the relationship between AR and TV in terms of time,

- their relationship in terms of display location,

- the influence of the viewers' context on the experience, and

- the level of editorial control on the experience.

The dimensions are described in terms of their 'aspects'. Table 3 presents these dimensions and their aspects.

\subsection{Abstraction}

We build on top of Jones et al.'s abstraction dimension [25], and extend it to describe the semantic relationship between AR and

\footnotetext{
${ }^{6}$ https://en.wikipedia.org/wiki/Photogrammetry
}

TV. We consider the 'wholeness' and the 'completeness' of the experience to be the key deciding factors. In other words, either one or both components (AR and TV) are independently complete and meaningful, or the completeness of the experience relies on the presence of one or both components.

Independent. This is when AR and TV experiences are independently complete. They may, however, be semantically related, for instance because they both originate from the same idea or are both based on the same script. This approach can be used to create self-contained mixed and augmented reality experiences based on a TV show [18]. ${ }^{7}$

Dependent. In this case the 'wholeness' of the overall experience depends on the existence of both components. For instance, the content created in a virtual studio setting often requires the existence of both elements; removing a virtual presenter [40] from the TV show would make the entire experience meaningless. This type of abstraction can be used to create novel engaging experiences across TV and AR [45].

Additional AR. There are experiences that mix AR and TV in such a way that the TV experience remains meaningful after elimination of AR. In these cases, AR is dependent on the TV content while $\mathrm{TV}$ is meaningful independently. In such cases AR is often used to enhance the TV viewing experience by providing extra features or novel interaction techniques [3,58-60].

Additional TV. We found no instances where the experience remains meaningful once the TV component is eliminated. ${ }^{8}$

\subsection{Interaction}

Interactions enable the viewers to dynamically change an aspect of their experience. We found three types of interactions in the literature that reflect the goal of the designer: display-level, structurelevel, and content-level.

Display-level. These interactions enable the viewers to perform tasks such as: changing the programme, resizing or repositioning the content, etc. $[3,58,59]$. A more advanced display-level interaction may enable the viewers to change camera angles [21].

Structure-level. This level of interaction breaks the linear structure and enables the viewers to only view the elements that interest them in any order [4]. This is akin to the concept of hyperlinks in the textual paradigm.

Content-level. This level of interaction is akin to that in the gaming paradigm which enables direct manipulation of the elements within the content, such as characters and objects $[18,26,29,40,45$, $52,55]$

\subsection{Time}

Traditional television content is typically delivered on a timeline. Similarly, the presentation of AR content can be visualised on a timeline. When delivering experiences that mix AR with TV, researchers have often attempted to synchronise these distinct timelines [26,27].

\footnotetext{
${ }^{7}$ The BBC's Civilisations AR app and Pokémon Go are other such examples. ${ }^{8}$ Except when the two experiences are independent, or when AR is used to deliver TV content.
} 
From the point of view of the viewers AR and TV components can be viewed in three ways: continuous, intermittent, or asynchronous.

Continuous. Here, both components (AR and TV) are presented to the viewers at the same time [26,27] (Figure 3).

Intermittent. In this case, the experience consists of presenting content over TV and AR intermittently, for instance to mix an AR game with an educational TV programme [45] (Figure 4).

Asynchronous. There are cases where synchronisation of AR with TV is not the aim of the designer. Often, these are independent AR and TV experiences (see Abstraction) [18].

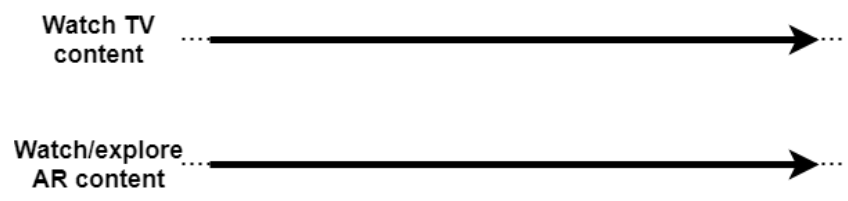

Figure 3: Visualisation of the continuous time.

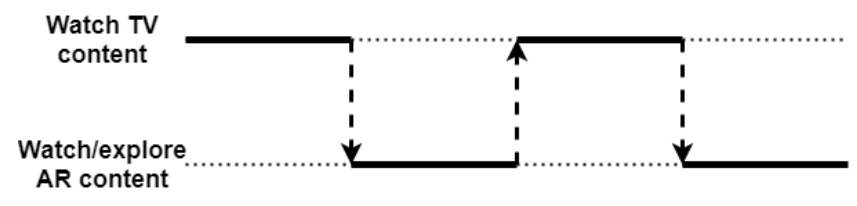

Figure 4: Visualisation of the intermittent time.

\subsection{Context}

Context reflects the designers' goals regarding the ways in which, if at all, the viewers' surrounding environment affects the experience. We present these in three classes of: people, place, and objects.

People. There are instances of mixing AR with TV that have been designed with multiple viewers in mind. For instance to enable friends and family members [3,31,45,52,58,60], or colleagues [22] to share aspects of an experience.

Transforming or adapting such experiences based on the presence of bystanders and passers-by, and the the primary viewers' relationship and interactions with them, may be a necessary step to make such experiences acceptable in public.

Place. In some experiences the content is anchored to specific physical locations and becomes available when the viewer reaches these locations [14,41,42].

Objects. In its simplest form, the system is aware of the existence and location of the TV screen. This enables AR content to be displayed in the area surrounding the TV [59]. Other implementations use knowledge of the surface colours and geometry, and apply radiometric compensation $[5,6,44]$ to adapt the appearance of the content to the viewers' environment [25].

\subsection{Display}

When combining TV and AR, content can be displayed in three ways: on the same display, on separate displays, or TV content presented using AR.

$A R$ and TV on the Same Display. Often the results of mixing AR with TV is displayed on the same display. Examples include the content created in a virtual studio setup $[15,40]$, or experiences that enable viewers to view or interact with additional content using $\operatorname{AR}[55,60]$.

$A R$ and TV on Separate Displays. This is akin to the use of second screens for viewing additional content while watching TV [17,34]. In the context of mixing AR with TV, the secondary display can be used to create novel TV viewing and cross-media experiences [26, $27,45,61]$.

$T V$ Viewing with $A R$. An AR display can be used to replace the TV screen in two ways: viewing of TV content presented in virtual screens $[41,42,59]$, and display of TV-like content beyond any visible rectangular frame [66]. While the former can be readily applied to existing TV content, the latter requires creation of content using technologies such as chroma-keying and volumetric capture.

\subsection{Editorial Control}

In a conventional TV programme, content creators often have control over two aspects of the viewers' experience: sequencing of media elements, and viewers' point of view. Mixing AR with TV enables new opportunities to delegate aspects of control back to the viewers.

Sequencing of Media Content. TV content is often created by editing shots and sequences in a specific way, dictated by the director's style which is influenced by existing conventions. Viewers of a TV programme typically have no control over the order in which various elements of the programme are presented to them. In this context AR can provide opportunities to allow the viewers a freeexploration style approach to viewing the content $[4,20,41,42,45]$.

Creating a successful experience may rely on the right combination of the two ends of this spectrum (fully edited vs fully exploratory), based on design intentions, genre, demographics of the viewers, etc.

Point of View. Traditional audio-visual media (e.g. film and TV) fix the viewers' POV on the screen. This allows the director to dynamically change the POV-based on their stylistic choices and existing conventions-to guide the viewers' gaze through the story.

AR can be used to enable the viewers to select from a few possible camera angles [21], or to provide a completely free-view point [27, 55].

While the affordances of a screen (e.g. a TV display) make it suitable for delivering content with a fixed POV, AR can be used to deliver content with a fixed POV [61], context-aware POV [66], viewers' choice of camera angle [21], or fully free POV [27].

\section{PATTERNS}

When mixing TV and AR, the dimensions can be combined to create unique patterns. A set comprised of the Cartesian product of all 
aspects of the dimensions results in 648 unique patterns. A closer look at this set, however, reveals that not all patterns are valid; Regarding the abstraction dimension (see Dimensions in Section 5), when creating independent AR and TV experiences there is no need for the two components to be synchronised. Conversely, dependent, additional $A R$, and additional $T V$ aspects of this dimension require a form of synchronisation between the TV and AR components. Eliminating the invalid patterns that combine these aspects reduces the number to 378 possible patterns.

While detailed enumeration and discussion of all possible patterns is outside the scope of this paper, we give examples of how to generate patterns and how to subsequently use them for design purposes. We suggest two methods for exploring the design space implied by the dimensions: sampling from the set of all possible patterns, and ideation cards.

\subsection{Sampling from the Set}

We present two random samples from the set generated by the Cartesian product of all dimensions (and their aspects) and explore the possibilities by writing two scenarios.

Sample 1.

- Abstraction: dependent

- Interaction: content-level

- Time: intermittent

- Display: separate displays

- Context: location-aware

- Editorial Control: exploratory AR

Scenario 1. Imagine a crime series where the story elements are divided and told across both TV and AR. While the established conventions of television are used to create content that the viewers watch on the TV, following the end of the episode, the viewers need to find a clue to help the detective solve a crime. For instance, the living room may be transformed into the crime scene, or the viewers may need to visit a local café to find the virtual clue using their smart phone. They can only watch the next episode after 'throwing' the clue back into the physical TV set.

Sample 2.

- Abstraction: independent

- Interaction: display-level

- Time: asynchronous

- Display: separate displays

- Context: location-aware

- Editorial Control: edited content

Scenario 2. Imagine in the previous crime scene a spin-off story is created for the AR platform. Hard-core fans of the show can experience these spin-offs of various characters in various locations. To view the content related to the backstory of a character that supposedly lives in London's East End, local fans can go to an old church in the area, find the specified bench and sit there next to the character and listen to them tell their backstory. The content is independent in the sense that it contains a stand-alone story and can even be understood by someone who does not watch the TV show. Interaction is display-level enabling the viewer to select content that they want to view.

\subsection{Ideation Cards}

Mixed reality card games have been used as tools to enable collaborative design in the creation of mixed reality games in a playful manner [62]. We suggest a similar approach to foster collaborative content creation for experiences that mix TV and AR.

This can bring a team of interdisciplinary professionals (e.g. producers, engineers, screenwriters, filmmakers, playwrights, animators, computer graphics artists, game designers, etc.) together in the ideation phase and enable them to explore the design space from various perspectives.

We suggest an iterative approach to the designing of the cards. For instance using colours to represent the dimensions (e.g. red for abstraction). Various aspects of each dimension can then be represented using various shades of those colours.

In an imaginary ideation meeting the cards can be used in the following way: One person is in charge of the cards. They separate the cards based on colours and go through each colour discussing the possibilities laid out by each dimension. For instance, regarding abstraction they can discuss the suitability of dependent, independent, additional $A R$, or additional $T V$ aspects in creating the experience. Each party can present opportunities and challenges that each aspect presents to the project from their point of view. For instance, the screenwriter and the playwright discuss various potentials regarding character development, action, and location of the story; the engineer, animator and computer graphic artist can provide informed suggestions on the capabilities and limitations of the technology; the producers can have input on budget and editorial aspects of the content; the filmmaker discusses various styles of the audio-visual presentation provided by each aspect; and the game designer can provide input on how various aspects of abstraction may influence their choice when later they have to decide on the aspects of the interaction dimension.

They go through each dimension, discussing their ideas. It may be necessary to do this iteratively before the design is finalised.

\section{DISCUSSION AND SAMPLE GUIDELINES}

\subsection{Production, Distribution, Consumption, and Method}

Broadcasters' efforts can typically be categorised in three phases as: production, distribution, and consumption. These three categories are reflected in the contributions of the papers in the survey.

- Production-oriented papers often focus on enhancing the tools and processes involved in capturing and producing TV content.

- Distribution-oriented papers attempt to improve on existing ways, or introduce novel ways of distributing the content.

- Consumption-oriented publications, focus on enhancing existing experiences, or creating novel viewing experiences.

We found papers with contributions regarding a method or an algorithm, in addition to (or instead of) directly contributing to the three aforementioned phases. Table 4 presents the papers and their contributions regarding these four categories.

The majority of the publications focus on either production or consumption with fewer papers focusing on distribution. The application of AR in production and consumption of TV content can 
Table 4: Summary of the 42 papers analyzed in terms of contribution areas. The majority of papers make contributions in only one broadcasting phase: production (pd.), distribution (dt.), or consumption (cs.). Some additionally provide methodological (md.) contributions (Maalej et al. [35] are novel in contributing only through their method rather than to any specific broadcasting activity phase).

\begin{tabular}{|c|c|c|c|c|}
\hline \multirow[t]{2}{*}{ Paper } & \multicolumn{4}{|c|}{ Contribution } \\
\hline & Pd. & Dt. & Cs. & Md. \\
\hline Ghellal \& Lindt (2008) [18] & - & - & $\checkmark$ & - \\
\hline Sitnik et al. (2008) [54] & $\checkmark$ & - & - & - \\
\hline Han et al. (2010) [21] & $\checkmark$ & $\checkmark$ & $\checkmark$ & $\checkmark$ \\
\hline $\mathrm{Li}(2010)[32]$ & $\checkmark$ & $\checkmark$ & $\checkmark$ & - \\
\hline Oyarzun et al. (2010) [40] & $\checkmark$ & - & $\checkmark$ & - \\
\hline Schiller et al. (2010) [51] & $\checkmark$ & - & - & $\checkmark$ \\
\hline Denia et al. (2011) [15] & $\checkmark$ & - & - & $\checkmark$ \\
\hline $\operatorname{Kim}(2011)[28]$ & $\checkmark$ & - & - & $\checkmark$ \\
\hline Yan \& Zhang (2011) [64] & $\checkmark$ & - & - & $\checkmark$ \\
\hline Cushen and Nixon (2012) [12] & - & - & - & $\checkmark$ \\
\hline Li et al. (2012) [33] & $\checkmark$ & - & - & $\checkmark$ \\
\hline Vatavu (2012) [58] & - & - & $\checkmark$ & $\checkmark$ \\
\hline Jones et al. (2013) [25] & - & - & $\checkmark$ & $\checkmark$ \\
\hline Vatavu (2013) [59] & - & - & $\checkmark$ & $\checkmark$ \\
\hline Gaspari et al. (2014) [13] & $\checkmark$ & - & - & - \\
\hline Gómez et al. (2014) [20] & - & - & $\checkmark$ & $\checkmark$ \\
\hline Hough et al. (2014) [23] & $\checkmark$ & - & - & - \\
\hline Kawakita \& Nakagava (2014) [26] & $\checkmark$ & $\checkmark$ & $\checkmark$ & $\checkmark$ \\
\hline Saito et al. (2014) [49] & - & - & - & $\checkmark$ \\
\hline Simsch \& Herder (2014) [53] & $\checkmark$ & - & - & $\checkmark$ \\
\hline Biblioni et al. (2015) [4] & $\checkmark$ & - & $\checkmark$ & - \\
\hline Maalej et al. (2015) [35] & - & - & - & $\checkmark$ \\
\hline Park \& Woo (2015) [41] & $\checkmark$ & $\checkmark$ & $\checkmark$ & $\checkmark$ \\
\hline Revelle et al. (2015) [45] & - & - & $\checkmark$ & $\checkmark$ \\
\hline Vatavu (2015) [60] & - & - & $\checkmark$ & $\checkmark$ \\
\hline Schreer et al. (2016) [52] & $\checkmark$ & $\checkmark$ & $\checkmark$ & $\checkmark$ \\
\hline Bailard et al. (2017) [3] & - & $\checkmark$ & $\checkmark$ & - \\
\hline Chandrasekan \& Kesavan (2017) [8] & - & $\checkmark$ & $\checkmark$ & - \\
\hline Kim et al. (2017) [29] & - & $\checkmark$ & $\checkmark$ & $\checkmark$ \\
\hline Lee et al. (2017) [31] & - & $\checkmark$ & $\checkmark$ & $\checkmark$ \\
\hline Park \& Woo (2017) [42] & - & - & $\checkmark$ & $\checkmark$ \\
\hline Rodrigues et al. (2017) [46] & - & - & $\checkmark$ & $\checkmark$ \\
\hline Sanna et al. (2017) [50] & $\checkmark$ & - & - & $\checkmark$ \\
\hline Sotelo et al. (2017) [55] & - & $\checkmark$ & $\checkmark$ & $\checkmark$ \\
\hline Cho et al. (2018) [9] & $\checkmark$ & - & - & - \\
\hline Debandi et al. (2018) [14] & - & - & $\checkmark$ & - \\
\hline Herder et al. (2018) [22] & $\checkmark$ & - & - & - \\
\hline Kawamura \& Otsuki (2018) [27] & $\checkmark$ & $\checkmark$ & - & - \\
\hline Kimura \& Rekimoto (2018) [30] & - & - & $\checkmark$ & $\checkmark$ \\
\hline Vinayagamoorthy et al. (2018) [61] & $\checkmark$ & - & $\checkmark$ & - \\
\hline Zhang \& Zhu (2018) [65] & $\checkmark$ & - & - & $\checkmark$ \\
\hline Zimmer et al. (2018) [66] & $\checkmark$ & - & $\checkmark$ & - \\
\hline
\end{tabular}

be understood in the context of reducing production costs, and creating novel experiences for the viewers, respectively. The limited number of contributions regarding distribution may be due to researchers' assumption that the content will be distributed using the Internet, as this has been the general tendency to distribute additional TV services.
There are, however, papers that cover all aspects of the broadcast chain $[21,26,32,41,52]$. For example, enabling viewers to participate in a pre-recorded quiz show [52] and broadcast the modified content to their friends. This suggests a shift in the viewers' role; from passively consuming content to actively creating new content-or manipulating existing content-and broadcasting it to their friends. Commercial availability of high quality tools to capture, manipulate and view content, may encourage more viewers to take part in this form of activity.

Design Guideline 1. When creating experiences for the hybrid medium, consider providing features that enable the viewers to manipulate existing content, or to create original content, that the viewers can subsequently distribute via the Internet.

\subsection{Viewers' Attention}

Attention can be seen as a limited resource and is commonly described using a 'spotlight' metaphor, where "a particular region of the visual scene" is selected "for more detailed processing" [16]. When content is delivered over two separate displays in continuous time (see Dimensions' Section), some viewers may experience confusion and a sense of 'missing out' [47]. Thoughtful orchestration of content and using appropriate cueing mechanisms-to orient [43] viewers' attention-can reduce this effect by reducing viewers' mental load.

Design Guideline 2. When creating content for the hybrid medium, if the content is delivered in continuous time over separate screens, consider using visual and auditory cues to guide the viewers' attention.

It may also be possible to use other approaches to reduce the viewers' mental load. We present them as guidelines 3 and 4 .

Design Guideline 3. If the content is being delivered over separate displays, consider using intermittent time instead of continuous time.

Design Guideline 4. When delivering AR content using a separate display in continuous time, visually limit the space where the AR content can appear (e.g. to the area surrounding the TV).

\subsection{Interdisciplinary Work}

A wide range of expertise and knowledge may be required to create a successful experience that mixes AR with TV.

In TV often the space of action is limited to a 2-D screen. In contrast, AR enables the action to take place anywhere in the viewers' environment. From the viewers' point of view AR may be more similar to theatre than TV. In addition, AR enables transforming the viewers' environment into a story-space (e.g. by adding objects from the story). This suggests that the knowledge and expertise of a set-designer may be useful. Further, the viewers can interact with objects and characters within the story-space, which suggests that the knowledge and expertise of a game designer may be useful.

Design Guideline 5. When creating content for the hybrid medium, consider using knowledge and expertise from a wide range of fields such as TV, gaming, theatre, set design, etc. 


\subsection{Dimensions' Aspects}

Abstraction, time, and display allow exactly one aspect to be selected per pattern. For instance, when combining TV with AR the relationship between the two can be either of independent, dependent, additional AR, or additional TV. However, interaction, context, and editorial control allow any number of aspects to be selected from each dimension (including no aspect at all). For instance, an experience-which mixes AR with TV-can allow display-level interaction as well as allowing content-level interaction. Conversely, it can be designed without any interactions at all. In cases where multiple aspects are allowed, designers can create richer experiences for the viewers by including more than one aspect per dimension.

Design Guideline 6. When considering dimensions that allow multiple aspects, consider implementing more than one aspect to create a richer experience for the viewers.

\subsection{Context-aware Experiences}

Experiences that mix AR with TV can be generated or adapted to take into account the viewers' context. So far the prototypes and scenarios use this dimension in a limited way and based on the available technology (e.g. anchoring video clips or 3-D models to a physical location). Generating ideas that take full advantage of this dimension may be used to direct the technology. For instance, adapting the content based on the viewers' relationship with bystanders or passers-by may be useful to avoid socially awkward situations.

\subsection{Meaningless Patterns}

There are patterns that combine aspects of dimensions in a way that the resulting design pattern is invalid. Some aspects of dimensions cannot fit in the same pattern with certain other aspects of other dimensions.

We eliminated 135 patterns that contained aspects of abstraction and time that made them unusable or illogical. A systematic exploration of the patterns can reduce the size of design space further.

\section{CONCLUSION}

The technology that enables the realisation of AR experiences for the public is maturing. However, creating successful AR experiences requires an understanding of opportunities and challenges that this medium provides [48]. In the context of broadcasting, individual efforts have been made to use AR's affordances to enhance or transform various aspects of the broadcast chain, although these efforts appear to be disjointed.

In this paper, our overall aim has been to provide an overview of the efforts in this field. This can help to identify opportunities for creating experiences that mix AR with TV. It can also highlight under-researched areas and guide future research in the field.

We have distilled information in the field-gathered from a representative sample of the literature-into an operable framework consisting of six primary dimensions of the design space-abstraction, interaction, time, display, context, and editorial control-and their respective aspects.
Researchers should be able to use the methods provided in this paper to systematically explore the design space as a way of generating guidelines.

Content creators and producers should be able to use these methods directly to make informed design decisions when creating experiences that mix AR with TV.

\section{ACKNOWLEDGMENTS}

Research reported in this paper was partially-funded by the UK EPSRC under grant numbers EP/R512394/1 and EP/N028228/1 (PACTMAN).

\section{REFERENCES}

[1] R. Azuma, Y. Baillot, R. Behringer, S. Feiner, S. Julier, and B. MacIntyre. 2001. Recent advances in augmented reality. IEEE Computer Graphics and Applications 21, 6 (Nov 2001), 34-47. https://doi.org/10.1109/38.963459

[2] Ronald T. Azuma. 1997. A Survey of Augmented Reality. Presence: Teleoperators and Virtual Environments 6, 4 (1997), 355-385. https://doi.org/10.1162/pres.1997. 6.4.355 arXiv:https://doi.org/10.1162/pres.1997.6.4.355

[3] Caroline Baillard, Matthieu Fradet, Vincent Alleaume, Pierrick Jouet, and Anthony Laurent. 2017. Multi-device Mixed Reality TV: A Collaborative Experience with Joint Use of a Tablet and a Headset. In Proceedings of the 23rd ACM Symposium on Virtual Reality Software and Technology (VRST '17). ACM, New York, NY, USA, Article 67, 2 pages. https://doi.org/10.1145/3139131.3141196

[4] Toni Bibiloni, Antoni Oliver, Cristina Manresa-Yee, and Javier Varona. 2015. Usability testing of a Hypervideo-based Interactive TV application. In VI International Conference on Interactive Digital TV IV Iberoamerican Conference on Applications and Usability of Interactive TV. 127.

[5] Oliver Bimber and Daisuke Iwai. 2008. Superimposing Dynamic Range. ACM Trans. Graph. 27, 5, Article Article 150 (Dec. 2008), 8 pages. https://doi.org/10. 1145/1409060.1409103

[6] Oliver Bimber and Ramesh Raskar. 2005. Spatial augmented reality: merging real and virtual worlds. AK Peters/CRC Press.

[7] Jay David Bolter, Richard Grusin, and Richard A Grusin. 2000. Remediation: Understanding new media. mit Press.

[8] Shivakumar Chandrasekaran and Umesh Kesavan. 2017. Augmented reality in broadcasting. In 2017 IEEE International Conference on Consumer Electronics-Asia (ICCE-Asia). IEEE, 81-83.

[9] Hyunwoo Cho, Sung-Uk Jung, Suwon Lee, Young-Suk Yoon, Sangheon Park, and Hyung-Keun Jee. 2018. ARStudio: AR Broadcasting System based on Multiple RGB-D Cameras. In 2018 International Conference on Information and Communication Technology Convergence (ICTC). IEEE, 1140-1143.

[10] Andy Cockburn, Amy Karlson, and Benjamin B. Bederson. 2009. A Review of Overview+detail, Zooming, and Focus+context Interfaces. ACM Comput. Surv. 41, 1, Article Article 2 (Jan. 2009), 31 pages. https://doi.org/10.1145/1456650.1456652

[11] Alan B. Craig. 2013. Understanding Augmented Reality: Concepts and Applications (1st ed.). Morgan Kaufmann Publishers Inc., San Francisco, CA, USA.

[12] George A Cushen and Mark S Nixon. 2012. Real-time semantic clothing segmentation. In International Symposium on Visual Computing. Springer, 272-281.

[13] Tiago De Gaspari, Antonio Carlos Sementille, Daniel Zuniga Vielmas, Ivan Abdo Aguilar, and João Fernando Marar. 2014. ARSTUDIO: A Virtual Studio System with Augmented Reality Features. In Proceedings of the 13th ACM SIGGRAPH International Conference on Virtual-Reality Continuum and Its Applications in Industry (VRCAI '14). ACM, New York, NY, USA, 17-25. https://doi.org/10.1145/ 2670473.2670491

[14] Federico Debandi, Roberto Iacoviello, Alberto Messina, Maurizio Montagnuolo, Federico Manuri, Andrea Sanna, and Davide Zappia. 2018. Enhancing cultural tourism by a mixed reality application for outdoor navigation and information browsing using immersive devices. In IOP Conference Series: Materials Science and Engineering, Vol. 364. IOP Publishing, 012048.

[15] Alberto Denia, Jose Ribelles, Angeles Lopez, and Óscar Belmonte. 2011. Low Cost Virtual Animation Effects for Sports Broadcasting: Mosaics, Flags and BigSized Flags. In 2011 International Conference on Computational Science and Its Applications. IEEE, 10-16.

[16] Jon Driver. 2001. A selective review of selective attention research from the past century. British fournal of Psychology 92, 1 (2001), 53-78.

[17] David Geerts, Rinze Leenheer, Dirk De Grooff, Joost Negenman, and Susanne Heijstraten. 2014. In Front of and behind the Second Screen: Viewer and Producer Perspectives on a Companion App. In Proceedings of the ACM International Conference on Interactive Experiences for TV and Online Video (TVX '14). Association for Computing Machinery, New York, NY, USA, 95-102. https: //doi.org/10.1145/2602299.2602312 
[18] Sabiha Ghellal and Irma Lindt. 2008. Interactive Movie Elements in a Pervasive Game. Personal Ubiquitous Comput. 12, 4 (Feb. 2008), 307-315. https://doi.org/ 10.1007/s00779-007-0186-8

[19] Simon Gibbs, Constantin Arapis, Christian Breiteneder, Vali Lalioti, Sina Mostafawy, and Josef Speier. 1998. Virtual studios: An overview. IEEE multimedia 5, 1 (1998), 18-35.

[20] David Gómez, Ana M Bernardos, and José R Casar. 2014. A system to enable level-of-detail mobile interaction with augmented media objects. In 2014 Eighth International Conference on Innovative Mobile and Internet Services in Ubiquitous Computing. IEEE, 346-351.

[21] Jungong Han, Dirk Farin, and Peter de With. 2010. A mixed-reality system for broadcasting sports video to mobile devices. IEEE MultiMedia 18, 2 (2010), 72-84.

[22] Jens Herder, Philipp Ladwig, Kai Vermeegen, Dennis Hergert, Florian Busch Kevin Klever, Sebastian Holthausen, and Bektur Ryskeldiev. 2018. Mixed Reality Experience-How to Use a Virtual (TV) Studio for Demonstration of Virtual Reality Applications. (2018).

[23] Gregory Hough, Ian Williams, and Cham Athwal. 2014. Measurements of live actor motion in mixed reality interaction. In 2014 IEEE International Symposium on Mixed and Augmented Reality (ISMAR). IEEE, 99-104.

[24] Simon Hudson and JR Brent Ritchie. 2006. Promoting destinations via film tourism: An empirical identification of supporting marketing initiatives. fournal of travel research 44, 4 (2006), 387-396.

[25] Brett R. Jones, Hrvoje Benko, Eyal Ofek, and Andrew D. Wilson. 2013. IllumiRoom Peripheral Projected Illusions for Interactive Experiences. In Proceedings of the SIGCHI Conference on Human Factors in Computing Systems (CHI '13). ACM, New York, NY, USA, 869-878. https://doi.org/10.1145/2470654.2466112

[26] Hiroyuki Kawakita and Toshio Nakagawa. 2014. Augmented TV: An augmented reality system for TV programs beyond the TV screen. In 2014 International Conference on Multimedia Computing and Systems (ICMCS). IEEE, 955-960.

[27] Yuki Kawamura and Kazuhiro Otsuki. 2018. 3D free-viewpoint augmented reality on a second display synchronized with a broadcast program. In Proceedings of the 4th International Conference on Communication and Information Processing. ACM, 273-277.

[28] Seoksoo Kim. 2011. Virtual studio system for augmented reality \& Chroma key processing. In 13th International Conference on Advanced Communication Technology (ICACT2011). IEEE, 762-765.

[29] Soonchoul Kim, Moonhyun Kim, Kuinam J Kim, Bumsuk Choi, and Jinwook Chung. 2017. A scheme of AR-based personalized interactive broadcasting service in terrestrial digital broadcasting system. Cluster Computing 20, 3 (2017), 2349-2354.

[30] Naoki Kimura and Jun Rekimoto. 2018. ExtVision: Augmentation of Visual Experiences with Generation of Context Images for a Peripheral Vision Using Deep Neural Network. In Proceedings of the 2018 CHI Conference on Human Factors in Computing Systems (CHI '18). ACM, New York, NY, USA, Article 427, 10 pages. https://doi.org/10.1145/3173574.3174001

[31] Hojun Lee, Gyutae Ha, Sangho Lee, and Shiho Kim. 2017. A mixed reality tele-presence platform to exchange emotion and sensory information based on MPEG-V standard. In 2017 IEEE Virtual Reality (VR). IEEE, 349-350.

[32] Ying Li. 2010. Augmented Reality for remote education. In 2010 3rd International Conference on Advanced Computer Theory and Engineering (ICACTE), Vol. 3. IEEE, V3-187.

[33] Yang Li, Dong-Xiao Li, Liang-Hao Wang, and Ming Zhang. 2012. Real time stereo rendering for augmented reality on 3DTV system. In 2012 International Conference on Systems and Informatics (ICSAI2012). IEEE, 2125-2129.

[34] Valentin Lohmüller and Christian Wolff. 2019. Towards a Comprehensive Definition of Second Screen. In Proceedings of Mensch Und Computer 2019 (MuC'19). Association for Computing Machinery, New York, NY, USA, 167-177. https://doi.org/10.1145/3340764.3340781

[35] Lotfi Maalej, Yosr Mlouhi, Imed Jabri, and Tahar Battikh. 2015. Artificial vision and augmented reality applied to the analysis of sports broadcast videos. In 2015 2nd World Symposium on Web Applications and Networking (WSWAN). IEEE, 1-9.

[36] Paul Milgram, Herman Colquhoun, et al. 1999. A taxonomy of real and virtua world display integration. Mixed reality: Merging real and virtual worlds 1, 1999 (1999), 1-26.

[37] Paul Milgram and Fumio Kishino. 1994. A taxonomy of mixed reality visual displays. IEICE TRANSACTIONS on Information and Systems 77, 12 (1994), 13211329.

[38] Paul Milgram, Haruo Takemura, Akira Utsumi, and Fumio Kishino. 1995. Augmented reality: A class of displays on the reality-virtuality continuum. In Telemanipulator and telepresence technologies, Vol. 2351. International Society for Optics and Photonics, 282-292.

[39] David Moher, Alessandro Liberati, Jennifer Tetzlaff, and Douglas G Altman. 2009 Preferred reporting items for systematic reviews and meta-analyses: the PRISMA statement. Annals of internal medicine 151, 4 (2009), 264-269.

[40] David Oyarzun, Andoni Mujika, Aitor Álvarez, Aritz Legarretaetxeberria, Aitor Arrieta, and María del Puy Carretero. 2010. High-realistic and flexible virtual presenters. In International Conference on Articulated Motion and Deformable Objects. Springer, 108-117.
[41] Hyerim Park and Woontack Woo. 2015. Metadata design for location-based film experience in augmented places. In 2015 IEEE International Symposium on Mixed and Augmented Reality-Media, Art, Social Science, Humanities and Design. IEEE, $40-45$

[42] Hyerim Park and Woontack Woo. 2017. Metadata design for AR spacetelling experience using movie clips. In 2017 IEEE International Conference on Consumer Electronics (ICCE). IEEE, 388-391.

[43] Michael I Posner. 1980. Orienting of attention. Quarterly journal of experimental psychology 32, 1 (1980), 3-25.

[44] Ramesh Raskar, Greg Welch, Kok-Lim Low, and Deepak Bandyopadhyay. 2001. Shader lamps: Animating real objects with image-based illumination. In Rendering Techniques 2001. Springer, 89-102.

[45] Glenda Revelle, Emily Reardon, Kristin Cook, Lori Takeuchi, Rafael Ballagas, Koichi Mori, Hiroshi Horii, Hayes Raffle, Maria Sandberg, and Mirjana Spasojevic. 2015. Electric Agents: Combining Collaborative Mobile Augmented Reality and Web-Based Video to Reinvent Interactive Television. Comput. Entertain. 12, 3, Article 1 (Feb. 2015), 21 pages. https://doi.org/10.1145/2702109.2633413

[46] Eduardo Rodrigues, Lucas Silva Figueiredo, Lucas Maggi, Edvar Neto, Layon Tavares Bezerra, João Marcelo Teixeira, and Veronica Teichrieb. 2017. Mixed Reality TVs: Applying Motion Parallax for Enhanced Viewing and Control Experiences on Consumer TVs. In 2017 19th Symposium on Virtual and Augmented Reality (SVR). IEEE, 319-330.

[47] Pejman Saeghe, Sarah Clinch, Bruce Weir, Maxine Glancy, Vinoba Vinayagamoorthy, Ollie Pattinson, Stephen Robert Pettifer, and Robert Stevens. 2019. Augmenting Television With Augmented Reality. In Proceedings of the 2019 ACM International Conference on Interactive Experiences for TV and Online Video (TVX '19). ACM, New York, NY, USA, 255-261. https://doi.org/10.1145/3317697.3325129

[48] Pejman Saeghe, Sarah Clinch, Bruce Weir, Steve Pettifer, and Robert Stevens. 2019. Augmented Reality Broadcasting: Challenges and Opportunities. In "Challenges Using Head-Mounted Displays in Shared and Social Spaces": Workshop at the ACM CHI Conference on Human Factors in Computing Systems. ACM.

[49] Hideo Saito, Toshihiro Honda, Yusuke Nakayama, and Francois De Sorbier. 2014. Camera pose estimation for mixed and diminished reality in FTV. In 2014 3DTVConference: The True Vision-Capture, Transmission and Display of 3D Video (3DTVCON). IEEE, 1-4.

[50] Andrea Sanna, Fabrizio Lamberti, Francesco De Pace, Roberto Iacoviello, and Paola Sunna. 2017. ARSSET: Augmented Reality Support on SET. In International Conference on Augmented Reality, Virtual Reality and Computer Graphics. Springer, 356-376.

[51] Ingo Schiller, Bogumil Bartczak, Falko Kellner, and Reinhard Koch. 2010. Increasing Realism and Supporting Content Planning for Dynamic Scenes in a Mixed Reality System incorporating a Time-of-Flight Camera. FVRB-fournal of Virtual Reality and Broadcasting 7, 4 (2010).

[52] O Schreer, W Waizenegger, W Fernando, H Kodikara Arachchi, A Oehme, A Smolic, B Yargicoglu, A Akman, and U Curjel. 2016. Mixed reality technologies for immersive interactive broadcast. (2016)

[53] Jonathan Simsch and Jens Herder. 2014. SpiderFeedback: Visual Feedback for Orientation in Virtual TV Studios. In Proceedings of the 11th Conference on Advances in Computer Entertainment Technology (ACE '14). ACM, New York, NY, USA, Article 12, 8 pages. https://doi.org/10.1145/2663806.2663830

[54] Robert Sitnik, Slawomir Pasko, Maciej Karaszewski, and Marcin Witkowski. 2008. Internet virtual studio: low-cost augmented reality system for WebTV. In The Engineering Reality of Virtual Reality 2008, Vol. 6804. International Society for Optics and Photonics, 68040E

[55] Rafael Sotelo, Jose Joskowicz, and Nicolás Rondan. 2017. Experiences on hybrid television and augmented reality on ISDB-T. In 2017 IEEE International Symposium on Broadband Multimedia Systems and Broadcasting (BMSB). IEEE, 1-5.

[56] Maximilian Speicher, Brian D. Hall, and Michael Nebeling. 2019. What is Mixed Reality? In Proceedings of the 2019 CHI Conference on Human Factors in Computing Systems (CHI '19). ACM, New York, NY, USA, Article 537, 15 pages. https: //doi.org/10.1145/3290605.3300767

[57] Ivan E. Sutherland. 1968. A Head-mounted Three Dimensional Display. In Proceedings of the December 9-11, 1968, Fall foint Computer Conference, Part I (AFIPS '68 (Fall, part I)). ACM, New York, NY, USA, 757-764. https://doi.org/10.1145/ 1476589.1476686

[58] Radu-Daniel Vatavu. 2012. Point \& click mediated interactions for large home entertainment displays. Multimedia Tools and Applications 59, 1 (2012), 113-128.

[59] Radu-Daniel Vatavu. 2013. There's a World Outside Your TV: Exploring Interactions Beyond the Physical TV Screen. In Proceedings of the 11th European Conference on Interactive TV and Video (EuroITV '13). ACM, New York, NY, USA, 143-152. https://doi.org/10.1145/2465958.2465972

[60] Radu-Daniel Vatavu. 2015. Audience Silhouettes: Peripheral Awareness of Synchronous Audience Kinesics for Social Television. In Proceedings of the ACM International Conference on Interactive Experiences for TV and Online Video (TVX '15). ACM, New York, NY, USA, 13-22. https://doi.org/10.1145/2745197.2745207

[61] Vinoba Vinayagamoorthy, Maxine Glancy, Paul Debenham, Alastair Bruce, Christoph Ziegler, and Richard Schäffer. 2018. Personalising the TV Experience with Augmented Reality Technology: Synchronised Sign Language Interpretation. 
In Proceedings of the 2018 ACM International Conference on Interactive Experiences for TV and Online Video (TVX '18). ACM, New York, NY, USA, 179-184. https://doi.org/10.1145/3210825.3213562

[62] Richard Wetzel, Tom Rodden, and Steve Benford. 2017. Developing ideation cards for mixed reality game design. Transactions of the Digital Games Research Association 3, 2 (2017)

[63] Claes Wohlin. 2014. Guidelines for Snowballing in Systematic Literature Studies and a Replication in Software Engineering. In Proceedings of the 18th International Conference on Evaluation and Assessment in Software Engineering (EASE '14). ACM, New York, NY, USA, Article 38, 10 pages. https://doi.org/10.1145/2601248.2601268
[64] Yongxin Yan and Xiaolei Zhang. 2011. Research and analysis of the Virtual Reality with FLARToolKit. In 2011 International Conference on Mechatronic Science, Electric Engineering and Computer (MEC). IEEE, 1614-1617.

[65] Yanxiang Zhang and Ziqiang Zhu. 2018. Live Mixed Reality Video Production for Educational Presentation. In International Conference on Augmented Reality, Virtual Reality and Computer Graphics. Springer, 363-372.

[66] Christian Zimmer, Nanette Ratz, Michael Bertram, and Christian Geiger. 2018. War Children: Using AR in a Documentary Context. In 2018 IEEE International Symposium on Mixed and Augmented Reality Adjunct (ISMAR-Adjunct). IEEE, 390-394. 